\title{
UNIFORM ASYMPTOTIC APPROXIMATIONS FOR THE MEIXNER-SOBOLEV POLYNOMIALS
}

\author{
S. FARID KHWAJA \\ School of Mathematics, King's Buildings, \\ The University of Edinburgh, Edinburgh EH9 3JZ, UK \\ S.F.Khwaja@sms.ed.ac.uk \\ A. B. OLDE DAALHUIS \\ Maxwell Institute and School of Mathematics, King's Buildings, \\ The University of Edinburgh, Edinburgh EH9 3JZ, UK \\ A.OldeDaalhuis@ed.ac.uk \\ Received (Day Month Year) \\ Revised (Day Month Year)
}

\begin{abstract}
We obtain uniform asymptotic approximations for the monic Meixner-Sobolev polynomials $S_{n}(x)$. These approximations for $n \rightarrow \infty$, are uniformly valid for $x / n$ restricted to certain intervals, and are in terms of Airy functions. We also give asymptotic approximations for the location of the zeros of $S_{n}(x)$, especially the small and the large zeros are discussed. As a limit case we also give a new asymptotic approximation for the large zeros of the classical Meixner polynomials.

The method is based on an integral representation in which a hypergeometric function appears in the integrand. After a transformation the hypergeometric functions can be uniformly approximated by unity, and all that remains are simple integrals for which standard asymptotic methods are used. As far as we are aware, this is the first time that standard uniform asymptotic methods are used for the Sobolev-class of orthogonal polynomials.
\end{abstract}

Keywords: Meixner-Sobolev polynomials, zeros, uniform asymptotic approximations.

Mathematics Subject Classification 2000: 33C47, 33C10, 30E15, 41A60, 30C15.

\section{Introduction}

The monic Meixner-Sobolev polynomials $S_{n}(x)$ are orthogonal with respect to the discrete inner product

$$
(p, q)_{S}=\sum_{k=0}^{\infty}\{p(k) q(k)+\lambda \Delta p(k) \Delta q(k)\} \frac{c^{k}(\beta)_{k}}{k !},
$$

where $0<c<1, \beta>0, \lambda \geqslant 0$, and $\Delta$ is the usual forward difference operator defined by $\Delta p(k)=p(k+1)-p(k)$. When $\lambda=0$ the Meixner-Sobolev polynomials reduce to the classical Meixner polynomials. These polynomials were introduced in [1], 
and a recurrence relation involving $S_{n}, S_{n-1}$ and 2 classical Meixner polynomials are given in [2] and [7]. This recurrence relation is very useful for generating the polynomials. The large $n$ asymptotics in [2] is for non-oscillatory regions.

In this paper we give large $n$ asymptotic approximations that are valid on the real $x$ axis, hence, they include the oscillatory region $0 \leqslant x / n<\frac{1+\sqrt{c}}{1-\sqrt{c}}$. The starting point is the generating function given in [7]:

$$
G(t)=\sum_{n=0}^{\infty} S_{n}(x) t^{n}
$$

where

$$
G(t)=\frac{1}{1-t} \frac{\left(1-\frac{t}{a}\right)^{-x-\beta+\gamma+1}}{\left(1-\frac{t}{a c}\right)^{-x}(1-a c t)^{\gamma}}{ }_{2} F_{1}\left(\begin{array}{c}
-x, \gamma \\
\beta-1
\end{array} ; z(t)\right)
$$

in which

$$
z(t)=\frac{-t(1-c)\left(1-a^{2} c\right)}{(1-a c t)(a c-t)}, \quad \Longrightarrow \quad 1-z(t)=\frac{(1-a t)\left(1-\frac{t}{a}\right)}{(1-a c t)\left(1-\frac{t}{a c}\right)},
$$

and

$$
\gamma=\frac{(1-a)(\beta-1)}{1-a^{2} c}, \quad a=\frac{1+\eta c-\sqrt{(1+\eta c)^{2}-4 c}}{2 c}
$$

where $\eta=1+\lambda\left(1-\frac{1}{c}\right)^{2}>1$. Note that $0<a<1$ and that $c a^{2}-(1+\eta c) a+1=0$. In (1.3) the function ${ }_{2} F_{1}\left(\begin{array}{c}a, b \\ c\end{array} ; t\right)$ is the standard Gauss hypergeometric function. (See [8].) Note that the definition of $\gamma$ differs from the one used in [7].

From the generating function $G(t)$ we will obtain an integral representation for $S_{n}(x)$. The integrand will involve the Gauss hypergeometric function, and it seems not easy to obtain asymptotic approximations. However, we will use some of the linear transformations for hypergeometric functions, to express $S_{n}(x)$ as a sum of 3 integrals in which the hypergeometric functions can be approximated uniformly by unity. Hence, the result is a simple integral approximation where standard methods can be used.

The structure of the paper is as follows. In $\S 2$, we study the asymptotics as $n \rightarrow \infty$ and $x$ is bounded. The analysis is based on Darboux's method. We will obtain simple asymptotic approximations, and observe that the small zeros of $S_{n}(x)$ are located at $x=0,1,2, \cdots$, with an exponentially small error.

In $\S 3$ we use several transformations to obtain an integral representation that can be used for the uniform asymptotic approximations. We discuss the location of the saddle points of the phase functions of the integrals. When $x / n=Y_{ \pm}$, where

$$
Y_{-}=\frac{1-\sqrt{c}}{1+\sqrt{c}}, \quad \text { and } \quad Y_{+}=\frac{1+\sqrt{c}}{1-\sqrt{c}},
$$

these saddle points will coalesce, and Airy functions are needed in the uniform asymptotic approximations. 
The multi-valuedness of the hypergeometric functions is discussed in $\S 4$, and we conclude that along the contours of integration of the integrals given in $\S 3$, the hypergeometric functions can be approximated uniformly by unity.

In the following 4 sections, we discuss the uniform asymptotic approximations. Only one saddle point dominates in the case $Y_{-}<x / n<Y_{+}$and a simple saddle point method approximation is given in $\S 5$, where we also give a relative simple formula for the location of the zeros.

The cases $0 \leqslant x / n<Y_{-}, 0<x / n<Y_{+}$and $Y_{-}<x / n$ are discussed in the next 3 sections. In the first case, we give a uniform asymptotic approximation in terms of a gamma function, and in the other 2 cases, Airy functions are needed in the asymptotic approximations. The later case also gives information about the location of the large zeros of $S_{n}(x)$, which are discussed in the final section. Our three term asymptotic approximation for the large zeros is in terms of the zeros of the Airy function $\operatorname{Ai}(z)$. When we let $\lambda \rightarrow 0$, that is, $a \rightarrow 1$, we obtain a three term asymptotic approximation for the large zeros of the classical Meixner polynomials, and our result agrees with [6], in which a two term asymptotic approximation is given. The additional term in our approximation is surprisingly simple.

In figure 1 we display the graph of

$$
\frac{\frac{2}{3} n S_{n}(x)}{\frac{\operatorname{erfc}\left(20\left(Y_{-}-y\right)\right) \Gamma(x+1)}{\sqrt{n} a^{n} c^{(n+x) / 2} \Gamma(x+\gamma+1)}+\frac{\operatorname{erfc}\left(20\left(y-Y_{-}\right)\right) \Gamma(x+1) \Gamma(n-x-\gamma)}{n !(a c)^{n}(1-c)^{x}}} .
$$

With this rescaling the function is $O(1)$ on the whole interval. It clearly displays the oscillatory region $x \in\left[0, n Y_{+}\right]$, and the dramatic change near $x \approx n Y_{-}$.

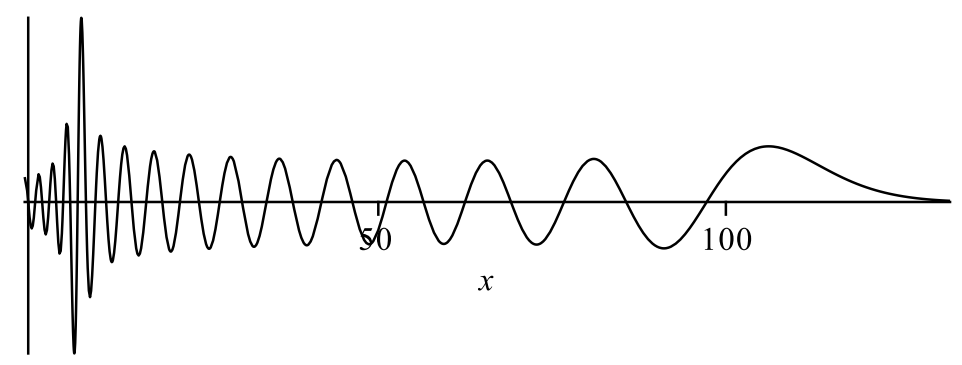

Fig. 1. The graph of a rescaled version of $S_{30}(x)$, where $a=\frac{5}{6}, c=\frac{1}{3}$ and $\beta=\frac{9}{8}$. Note the dramatic changes at $x=n Y_{-} \approx 8$ and at $x=n Y_{+} \approx 112$. 


\section{Large $n$ and fixed $x$ asymptotics}

For the large $n$ asymptotics, we will study the singularities of $G(t)$ in the complex $t$ plane. These are at

$$
a c, a, 1,1 / a, 1 / a c, \text { with } 0<a c<a<1<1 / a<1 / a c .
$$

Hence, the singularity that is nearest to the origin is $t=a c$. For an asymptotic expansion that holds as $n \rightarrow \infty$ and $a, c, \beta$ and $x$ fixed, we need the local expansion of $G(t)$ at this singularity. It is convenient to start with the identity

$$
G(t)=G_{1}(t)+G_{2}(t)
$$

(use (15.8.2) in [8]), where

$$
G_{1}(t)=K_{1} \frac{t^{\gamma-\beta+1}(1-a t)^{x+\beta-\gamma-1}}{(1-t)(1-a c t)^{x+\gamma}}{ }_{2} F_{1}\left(\begin{array}{c}
1-\gamma, \beta-\gamma-1 \\
1-\gamma-x
\end{array} ; \frac{1}{z(t)}\right)
$$

with

$$
K_{1}=\left((1-c)\left(\frac{1}{a c}-a\right)\right)^{\gamma-\beta+1} \frac{\Gamma(\beta-1) \Gamma(x+\gamma)}{\Gamma(\gamma) \Gamma(x+\beta-1)}
$$

and

$$
G_{2}(t)=K_{2} \frac{t^{-\gamma}\left(1-\frac{t}{a}\right)^{-x-\beta+\gamma+1}}{(1-t)\left(1-\frac{t}{a c}\right)^{-x-\gamma}} 2 F_{1}\left(\begin{array}{c}
\gamma, 2+\gamma-\beta \\
1+\gamma+x
\end{array} ; \frac{1}{z(t)}\right)
$$

with

$$
K_{2}=\left((1-c)\left(\frac{1}{a c}-a\right)\right)^{-\gamma} \frac{\Gamma(\beta-1) \Gamma(-x-\gamma)}{\Gamma(\beta-\gamma-1) \Gamma(-x)} .
$$

The function $G_{1}(t)$ has no singularity at $t=a c$. Hence, the main contributions to the large $n$ asymptotics will come from $G_{2}(t)$. We expand

$$
G_{2}(t)=K_{2} \sum_{m=0}^{\infty} b_{m}\left(1-\frac{t}{a c}\right)^{m+x+\gamma}
$$

with

$$
b_{0}=\frac{(1-c)^{-x-\beta+\gamma+1}(a c)^{-\gamma}}{1-a c},
$$

and obtain via Darboux's method (see $\S 2.10($ iv) in [10])

$$
\begin{aligned}
S_{n}(x) & \sim K_{2} \sum_{m=0}^{\infty} \frac{b_{m}}{2 \pi i} \oint_{\{0\}} \frac{\left(1-\frac{t}{a c}\right)^{m+x+\gamma}}{t^{n+1}} d t \\
& \sim K_{2} \frac{(a c)^{-n}}{n !} \sum_{m=0}^{\infty} b_{m}(-m-\gamma-x)_{n},
\end{aligned}
$$

as $n \rightarrow \infty$, where the Pochhammer symbol is $(z)_{n}=z(z+1)(z+2) \cdots(z+n-1)$. 
Just to illustrate: Taking $a=\frac{5}{6}, c=\frac{1}{3}$ and $\beta=\frac{9}{8}$, then $S_{50}(1.5)=3.256665 \times$ $10^{23}$ and taking 4 terms on the right-hand side of (2.9) gives us the approximation $3.256592 \times 10^{23}$. Hence, the relative error is 0.000023 .

It follows from the gamma function $\Gamma(-x)$ in $(2.6)$ that $S_{n}(x)$ has zeros at approximately $x=m$, where $m$ is a bounded nonnegative integer. In fact, since $K_{2}$ multiplies all terms in (2.9) and the contribution of $G_{1}(t)$ to the asymptotics of $S_{n}(x)$ will be exponentially small, it follows from Theorem 1 in [5], that the small zeros are located approximately at $x=m$ with an exponentially small error. In [6] the authors make the same observation for the small zeros of the classical Meixner polynomials.

\section{Large $n$ and $x$}

Our analysis will be based on the observation that (see $\S 15.12(i i)$ in [8])

$$
{ }_{2} F_{1}\left(\begin{array}{c}
A, B \\
x+C
\end{array} ; z\right)=1+O(1 / x), \quad x \rightarrow \infty, \quad|\operatorname{ph}(1-z)|<\pi .
$$

We will use this result for $x \rightarrow+\infty$. The result even holds for $x \rightarrow \infty$ in the sector $|\operatorname{ph} x| \leqslant \frac{1}{2} \pi-\varepsilon$, (where $\varepsilon$ is an arbitrary small positive constant), with the same restriction on $z$. For larger sectors in the complex $x$-plane the $z$-region of validity will be smaller.

The hypergeometric function in the right-hand side of (2.5) is already of the form (3.1). For the one in the representation (2.3) of $G_{1}(t)$ we need one more transformation.

Note that the function $G_{1}(t)$ is analytic at $t=a c$. For that function we push $t$ to $a c<\Re t<a$, and we use the transformation

$$
G_{1}(t)=G_{3}(t)-G_{4}(t),
$$

(combine (15.8.4) with (15.8.1) in [8]), where

$$
G_{3}(t)=K_{3} \frac{t^{\gamma-\beta+1}(1-a t)^{x+\beta-\gamma-1}}{(1-t)(1-a c t)^{x+\gamma}}{ }_{2} F_{1}\left(\begin{array}{c}
\beta-\gamma-1,1-\gamma \\
x+\beta-\gamma
\end{array} ; 1-\frac{1}{z(t)}\right)
$$

with

$$
K_{3}=\left((1-c)\left(\frac{1}{a c}-1\right)\right)^{\gamma-\beta+1} \frac{\Gamma(\beta-1) \Gamma(x+1)}{\Gamma(\gamma) \Gamma(x+\beta-\gamma)}
$$

and

$$
G_{4}(t)=K_{4} \frac{t^{-\gamma}\left(1-\frac{t}{a}\right)^{-x-\beta+\gamma+1}}{(1-t)\left(\frac{t}{a c}-1\right)^{-x-\gamma} 2} F_{1}\left(\begin{array}{c}
\gamma, 2+\gamma-\beta \\
1+\gamma+x
\end{array} ; \frac{1}{z(t)}\right)
$$

with

$$
K_{4}=\left((1-c)\left(\frac{1}{a c}-1\right)\right)^{-\gamma} \frac{\Gamma(\beta-1) \Gamma(x+1) \Gamma(-x-\gamma)}{\Gamma(\gamma) \Gamma(1-\gamma) \Gamma(\beta-\gamma-1)}
$$


We observe that $G_{2}(t)$ and $G_{4}(t)$ are in terms of the same hypergeometric function, but that in $G_{2}(t)$ we start at $0<t<a c$, and in $G_{4}(t)$ we consider $a c<t<a$. Combining these two functions we obtain

$$
G_{ \pm}(t)=G_{2}(t)-G_{4}(t)=e^{\mp x \pi i} K\left(\frac{\frac{t}{a c}-1}{1-\frac{t}{a}}\right)^{x} g(t)_{2} F_{1}\left(\begin{array}{c}
\gamma, 2+\gamma-\beta \\
1+\gamma+x
\end{array} ; \frac{1}{z(t)}\right)
$$

valid in the half-plane $\pm \Im(t)>0$, where

$$
K=\left((1-c)\left(\frac{1}{a c}-a\right)\right)^{-\gamma} \frac{\Gamma(\beta-1) \Gamma(x+1)}{\Gamma(\beta-\gamma-1) \Gamma(x+1+\gamma)},
$$

and

$$
g(t)=\frac{t^{-\gamma}\left(1-\frac{t}{a}\right)^{\gamma-\beta+1}}{(1-t)\left(\frac{t}{a c}-1\right)^{-\gamma}}
$$

Combining these results we obtain

$$
\begin{aligned}
S_{n}(x) & =\frac{1}{2 \pi i} \oint_{\{0\}} \frac{G(t)}{t^{n+1}} d t \\
& =\frac{1}{2 \pi i} \int_{\mathcal{C}_{3}} \frac{G_{3}(t)}{t^{n+1}} d t+\frac{1}{2 \pi i} \int_{\mathcal{C}_{+}} \frac{G_{+}(t)}{t^{n+1}} d t-\frac{1}{2 \pi i} \int_{\mathcal{C}_{-}} \frac{G_{-}(t)}{t^{n+1}} d t
\end{aligned}
$$

where $\mathcal{C}_{3}$ is for the moment a vertical contour that crosses the real $t$-axis in the interval $(a c, a)$ and $\mathcal{C}_{ \pm}$is a contour that emanates from $t=a c$ and goes to infinity in the upper/lower half-plane. Again, the integrands in (3.10) have singularities at the points mentioned in (2.1), and possibly a branch-point at $t=0$.

From here onwards, we will take

$$
x=n y, \text { where } y>0, \text { bounded. }
$$

We will now replace the hypergeometric functions by unity, Hence, the phasefunction for integrand $G_{3}(t)$ is $f_{3}(t)$ and for integrands $G_{ \pm}(t)$ it is $f(t)$, where

$$
f_{3}(t)=\ln (t)+y \ln \left(\frac{1-a c t}{1-a t}\right), \quad f(t)=\ln (t)+y \ln \left(\frac{1-\frac{t}{a}}{\frac{t}{a c}-1}\right) .
$$

The saddle-points are located at

$$
\begin{aligned}
S p_{3 \pm} & =\frac{1}{2 a c}\left(y(c-1)+c+1 \pm \sqrt{(y(c-1)+c+1)^{2}-4 c}\right) \\
S p_{ \pm} & =\frac{a}{2}\left(y(c-1)+c+1 \pm \sqrt{(y(c-1)+c+1)^{2}-4 c}\right)
\end{aligned}
$$

Note that the saddle-points 'coalesce' when $y^{2}+2 y \frac{c+1}{c-1}+1=0$, that is, when $y=Y_{ \pm}$, where $Y_{ \pm}$are defined in (1.6).

One very useful observation: let

$$
\cos \theta=\frac{1+c-(1-c) y}{2 \sqrt{c}}, \quad \text { then } \quad S p_{ \pm}=a \sqrt{c} e^{ \pm \theta i}, \quad S p_{3 \pm}=\frac{1}{a \sqrt{c}} e^{ \pm \theta i}
$$


It follows from these representations that for $0<y<Y_{-}$, we have

$$
a c<S p_{+}<a \sqrt{c}<S p_{-}<a<1<\frac{1}{a}<S p_{3+}<\frac{1}{a \sqrt{c}}<S p_{3-}<\frac{1}{a c},
$$

(compare (6.1)), for $Y_{-} \leqslant y \leqslant Y_{+}$we have $\left|S p_{ \pm}\right|=a \sqrt{c}<1<\left|S p_{3 \pm}\right|=1 /(a \sqrt{c})$, and finally for $y>Y_{+}$, we have

$$
S p_{-}<-a \sqrt{c}<S p_{+}, \quad S p_{3-}<\frac{-1}{a \sqrt{c}}<S p_{3+}, \quad \text { and } \quad S p_{3 \pm}<S p_{ \pm}<0
$$

(compare (8.1)). See figure 3, in which we indicate the location of the dominant saddles in the case $a=\frac{5}{6}, c=\frac{1}{3}$ and $\beta=\frac{9}{8}$.

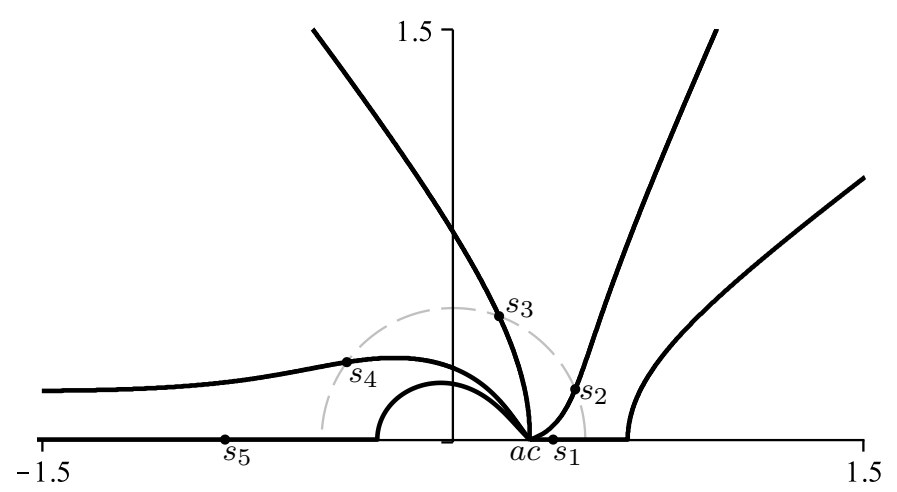

Fig. 2. Steepest descent contours $\mathcal{C}_{+}$in the cases $y=y_{j}$, where $y_{1}=\frac{1}{5}<Y_{-}, y_{2}=\frac{2}{5}, y_{3}=\frac{7}{5}$, $y_{4}=\frac{17}{5}$ and $y_{5}=4>Y_{+}$. The saddle points are located at $s_{j}$. Note that the contours emanate from $a c$ and that $s_{2}, s_{3}, s_{4}$ are located on the circle $|t|=a \sqrt{c}$.

\section{The multi-valuedness of the hypergeometric function}

The principal branch for the hypergeometric function ${ }_{2} F_{1}\left(\begin{array}{c}a, b \\ c\end{array} ; z\right)$ is $|\operatorname{ph}(1-z)|<$ $\pi$. Hence, the branch-cut is $z>1$. Thus, for the functions $G_{ \pm}(t)$ the $t$-branch-cuts are at points where $1 / z(t)>1$, that is, $0<z(t)<1$. The reader can check that this happens on the intervals $(-\infty, 0),(a, 1 / a)$ and on the unit circle $|t|=1$.

For the function $G_{3}(t)$ the $t$-branch-cuts are at points where $1-1 / z(t)>1$, that is, $z(t)<0$. This happens on the intervals $(0, a c)$ and $\left(\frac{1}{a c}, \infty\right)$.

If we continue to use the principal branches for the hypergeometric functions in the definition for $G_{ \pm}(t)$, then we have for $\Im t>0$

$$
\begin{aligned}
& G(t)=G_{+}(t)+G_{3}(t), \quad \text { for }|t|<1, \\
& G(t)=G_{+}(t)+e^{2(\beta-\gamma-1) \pi i} G_{3}(t), \quad \text { for }|t|>1 .
\end{aligned}
$$

Below we will see that the contributions of $G_{3}(t)$ are exponentially small compared with the contributions of $G_{ \pm}(t)$. It follows from (4.1) that we can use (3.1) for the 
hypergeometric functions in the right-hand side of (3.7) along the entire contour of integration.

\section{The case $Y_{-}<y<Y_{+}$}

Since

$$
\Re\left(f\left(S p_{ \pm}\right)-f_{3}\left(S p_{3 \pm}\right)\right)=\ln \left(a^{2} c\right)<0,
$$

it follows that the $G_{ \pm}(t)$-integrals in (3.10) dominate the asymptotics. For the $G_{+}(t)$ integral the steepest-descent contour of integration in the upper half $t$-plane, starts at $t=a c$ passes through the saddle-point at $t=S p_{+}$and goes to infinity. (See figure 3.) The contour of the $G_{-}(t)$ integral in the lower half plane is the complex conjugate of the contour in the upper half plane.

Let us study the integral

$$
\frac{1}{2 \pi i} \int_{\mathcal{C}_{+}} \frac{\widetilde{G_{+}}(t)}{t^{n+1}} d t
$$

where

$$
\frac{\widetilde{G_{+}}(t)}{t^{n+1}}=e^{-x \pi i} K e^{-n f(t)} g(t) / t,
$$

with $K, g(t)$ and $f(t)$ defined in (3.8), (3.9) and (3.12), respectively. Using the saddle point method (see $\S 2.4(\mathrm{iv})$ in [10]), the above integral, and hence $S_{n}(x)$ can be approximated by

$$
S_{n}(x) \sim 2 \Re\left(\frac{K e^{-x \pi i}\left(a \sqrt{c} e^{\theta i}\right)^{-n-\gamma}}{\left(1-a \sqrt{c} e^{\theta i}\right) \sqrt{2 \pi n\left(e^{2 \theta i}-1\right)}} \frac{\left(\frac{e^{\theta i}}{\sqrt{c}}-1\right)^{x+\gamma+1 / 2}}{\left(1-e^{\theta i} \sqrt{c}\right)^{x+\beta-\gamma-3 / 2}}\right),
$$

as $n \rightarrow \infty$, where $x=n y$ and $\theta$ is defined in (3.14).

The saddle-point approximation gives good results: Taking the same $a, c, \beta$ as before, and $y=0.7$ then $S_{50}(35)=-8.24876 \times 10^{22}$ and the dominant approximation from the 2 saddle points gives us $-8.27800 \times 10^{22}$. Hence, only one term gives us already a good approximation.

Regarding the zeros, it follows from (5.4) that these are approximately located where

$n(\theta+y(\pi-\theta))+(2 n y+\beta-1) \operatorname{ph}\left(1-\sqrt{c} e^{\theta i}\right)+\operatorname{ph}\left(1-a \sqrt{c} e^{\theta i}\right)=\left(k+\frac{1}{4}\right) \pi,(5.5)$

where $k$ is an integer. For example, one solution of $(5.5)$ is $y \approx 0.709082395$. Hence, approximately there should be a zero at $x=35.45412$. The 'exact' zero is located at $x=35.45469$.

The approximations in this section hold for $Y_{-}<y<Y_{+}$, that is, $0<\theta<\pi$. (Note the factor $e^{2 \theta i}-1$ in (5.4).) The saddle points $S p_{ \pm}=a \sqrt{c} e^{ \pm \theta i}$ coalesce when $\theta=0, \pi$. In the next sections we will obtain asymptotic approximations that hold in larger intervals, including the turning points at $\theta=0, \pi$. 


\section{The case $y \approx Y_{-}$}

We write (3.14) as

$$
\cos \theta=1-\frac{1-c}{2 \sqrt{c}}\left(y-Y_{-}\right), \quad \text { and take } \begin{aligned}
& y<Y_{-} \\
& y>Y_{-}
\end{aligned} \begin{array}{rr}
-\theta i>0, & \theta>0 .
\end{array}
$$

Hence, if $y \approx Y_{-}$then $\theta \approx 0$.

To obtain a uniform asymptotic approximation, we will use the cubic transformation suggested by Chester, Friedman and Ursell [4]

$$
f(t)=\frac{1}{3} u^{3}+\omega u+\psi .
$$

The right-hand side of (6.2) has saddle-points at $u=\mp i \sqrt{\omega}$, and we will insist that these correspond to $t=S p_{ \pm}=a \sqrt{c} e^{ \pm \theta i}$, respectively. This gives us the following two results

$$
\psi=\ln (a)+\frac{1}{2}(1+y) \ln (c)
$$

and

$$
-\frac{2}{3} i \omega^{3 / 2}=\theta i(1+y)+y \ln \left(\frac{e^{-\theta i}-\sqrt{c}}{e^{\theta i}-\sqrt{c}}\right) .
$$

The reader can check that for the right-hand side in (6.4), we have

$$
\theta i(1+y)+y \ln \left(\frac{e^{-\theta i}-\sqrt{c}}{e^{\theta i}-\sqrt{c}}\right) \sim-\frac{2}{3} i \frac{c^{-1 / 4}(1+\sqrt{c})^{2}}{\sqrt{1-c}}\left(y-Y_{-}\right)^{3 / 2},
$$

as $y \rightarrow Y_{-}$. Hence,

$$
\omega \sim \frac{c^{-1 / 6}(1+\sqrt{c})^{4 / 3}}{(1-c)^{1 / 3}}\left(y-Y_{-}\right), \quad \text { as } y \rightarrow Y_{-} .
$$

It is not difficult to show that on the interval $y \in\left(0, Y_{+}\right), \omega(y)$ is an increasing analytic function of $y$ with

$$
\omega(0)=-\left(\frac{3}{4} \ln (1 / c)\right)^{2 / 3}, \quad \omega\left(Y_{-}\right)=0, \quad \omega\left(Y_{+}\right)=\left(\frac{3 \pi \sqrt{c}}{1-\sqrt{c}}\right)^{2 / 3} .
$$

The local behaviour of transformation (6.2) near $t \approx a \sqrt{c} e^{\theta i}$ and $u \approx-i \sqrt{\omega}$ is

$$
\frac{-i e^{-2 \theta i} \sin \theta}{a^{2} \sqrt{c}(1-c) y}\left(t-a \sqrt{c} e^{\theta i}\right)^{2} \approx-i \sqrt{\omega}(u+i \sqrt{\omega})^{2},
$$

from which it follows that in the case $\omega<0$ we have to take $\sqrt{\omega}=i \sqrt{|\omega|}$.

Integral (5.2) becomes

$$
\frac{1}{2 \pi i} \int_{\mathcal{C}_{+}} \frac{\widetilde{G_{+}}(t)}{t^{n+1}} d t=\frac{e^{-x \pi i} K e^{-n \psi}}{2 \pi i} \int_{\infty e^{-2 \pi i / 3}}^{\infty} e^{-n\left(\frac{1}{3} u^{3}+\omega u\right)} g_{0}(u) d u,
$$

where

$$
g_{0}(u)=t^{-1} g(t) \frac{d t}{d u}=t^{-1} g(t) \frac{u^{2}+\omega}{f^{\prime}(t)} .
$$


The orientation of the $u$-integral in (6.9) follows when one takes the obvious squarehoods in (6.8). Note that from l'Hôpital's rule we obtain

It follows that

$$
\left.\frac{d t}{d u}\right|_{u= \pm i \sqrt{\omega}}=\sqrt{\frac{ \pm 2 i \sqrt{\omega}}{f^{\prime \prime}\left(a \sqrt{c} e^{\mp \theta i}\right)}} .
$$

$$
g_{0}( \pm i \sqrt{\omega})=\sqrt{\frac{\sqrt{w}(1-c) y}{\sqrt{c} \sin \theta}}\left(\frac{(1-c) y}{a c}\right)^{\gamma} \frac{\left(1-\sqrt{c} e^{\mp \theta i}\right)^{1-\beta}}{1-a \sqrt{c} e^{\mp \theta i}} .
$$

To obtain a uniform asymptotic approximation, we use Bleistein's Method [3] and substitute into (6.9)

$$
g_{0}(u)=p+q u+\left(u^{2}+\omega\right) h_{0}(u)
$$

where

$$
p=\frac{g_{0}(i \sqrt{\omega})+g_{0}(-i \sqrt{\omega})}{2}, \quad q=\frac{g_{0}(i \sqrt{\omega})-g_{0}(-i \sqrt{\omega})}{2 i \sqrt{\omega}}
$$

and obtain

$$
\begin{aligned}
\frac{1}{2 \pi i} \int_{\mathcal{C}_{+}} \frac{\widetilde{G_{+}}(t)}{t^{n+1}} d t \sim \frac{e^{-x \pi i} K e^{-n \psi}}{2 \pi i}\left(p \int_{\infty e^{-2 \pi i / 3}}^{\infty} e^{-n\left(\frac{1}{3} u^{3}+\omega u\right)} d u\right. \\
\left.\quad+q \int_{\infty e^{-2 \pi i / 3}}^{\infty} u e^{-n\left(\frac{1}{3} u^{3}+\omega u\right)} d u\right) .
\end{aligned}
$$

Using the change of variable $u=e^{-\pi i / 3} n^{-1 / 3} t$, we get

$$
\begin{array}{r}
\frac{1}{2 \pi i} \int_{\mathcal{C}_{+}} \frac{\widetilde{G_{+}}(t)}{t^{n+1}} d t \sim \frac{e^{-x \pi i} K e^{-n \psi}}{2 \pi i}\left(\frac{p e^{-\pi i / 3}}{n^{1 / 3}} \int_{\infty e^{-\pi i / 3}}^{\infty e^{\pi i / 3}} e^{\frac{1}{t^{3}}-\omega n^{2 / 3} e^{-\pi i / 3} t} d t\right. \\
\left.+\frac{q e^{-2 \pi i / 3}}{n^{2 / 3}} \int_{\infty e^{-\pi i / 3}}^{\infty e^{\pi i / 3}} t e^{\frac{1}{3} t^{3}-\omega n^{2 / 3} e^{-\pi i / 3} t} d t\right) .
\end{array}
$$

Which is equivalent to

$$
\begin{array}{r}
\frac{1}{2 \pi i} \int_{\mathcal{C}_{+}} \frac{\widetilde{G_{+}}(t)}{t^{n+1}} d t \sim e^{-x \pi i} K e^{-n \psi}\left(\frac{p e^{-\pi i / 3}}{n^{1 / 3}} \operatorname{Ai}\left(\omega e^{-\pi i / 3} n^{2 / 3}\right)\right. \\
\left.-\frac{q e^{-2 \pi i / 3}}{n^{2 / 3}} \operatorname{Ai}^{\prime}\left(\omega e^{-\pi i / 3} n^{2 / 3}\right)\right),
\end{array}
$$

as $n \rightarrow \infty$, where $\operatorname{Ai}(z)$ is the Airy function, see (9.5.4) in [9].

Since the polynomials are real-valued and the contribution of the $\mathcal{C}_{-}$will be just the complex conjugate of (6.17), we conclude that

$$
\begin{aligned}
S_{n}(x) \sim 2 \Re\left\{e^{-x \pi i} K e^{-n \psi}(\right. & \frac{p e^{-\pi i / 3}}{n^{1 / 3}} \operatorname{Ai}\left(\omega e^{-\pi i / 3} n^{2 / 3}\right) \\
& \left.\left.-\frac{q e^{-2 \pi i / 3}}{n^{2 / 3}} \mathrm{Ai}^{\prime}\left(\omega e^{-\pi i / 3} n^{2 / 3}\right)\right)\right\},
\end{aligned}
$$

as $n \rightarrow \infty$, uniformly for $y \in\left[\varepsilon, Y_{+}-\varepsilon\right]$, where $\varepsilon$ is a small positive constant. 


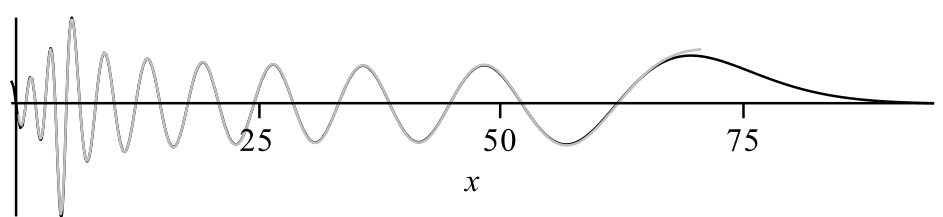

Fig. 3. The graph of a rescaled version of $S_{20}(x)$, where $a=\frac{5}{6}, c=\frac{1}{3}, \beta=\frac{9}{8}$ (black), and approximation (6.18) (grey). Note that only near $y=0$ and $y=Y_{+}$the difference is visible.

\section{The case $0 \leqslant y<Y_{-}$}

We will use the notation of the previous section. In $\S 2$, we dealt with the case of large $n$ and finite $x$, that is, $y \approx 0$, and in the previous section we covered the case $0<y<Y_{+}$. In the case of $0 \leqslant y<Y_{-}$, the active saddle-point of phase function $f(t)$ is at $t=S p_{+}$, and we have $a c \leqslant S p_{+}<a \sqrt{c}$. In integral representation (3.10) the main integrals are still the ones involving $G_{ \pm}$. We write

$$
\frac{1}{2 \pi i} \int_{\mathcal{C}_{ \pm}} \frac{G_{ \pm}(t)}{t^{n+1}} d t=\frac{e^{\mp x \pi i} K}{2 \pi i} \int_{\mathcal{C}_{ \pm}} e^{-n f(t)} \tilde{g}(t) d t
$$

where

$$
\tilde{g}(t)=\frac{t^{-\gamma-1}\left(1-\frac{t}{a}\right)^{\gamma-\beta+1}}{(1-t)\left(\frac{t}{a c}-1\right)^{-\gamma}}{ }_{2} F_{1}\left(\begin{array}{c}
\gamma, 2+\gamma-\beta \\
1+\gamma+x
\end{array} ; \frac{1}{z(t)}\right)
$$

Since $x$ appears in the bottom entry of the hypergeometric function, a possible large value for $x$ has only a positive influence in the expansion of this function near $t=S p_{+}$.

Note that as $y \rightarrow 0$, the saddle-point of phase function $f(t)$ at $t=S p_{+}$coalesces with the branch-point at $t=a c$. The local behaviour of $f(t)$ near $t=a c$ is

$$
f(t) \sim-y \ln \left(\frac{t}{a c}-1\right)+\ln (a c)+y \ln (1-c), \quad \text { as } t \rightarrow a c .
$$

For these reasons, we choose the transformation

$$
f(t)=u-\alpha \ln (u)+\ln (a c)+y \ln (1-c) .
$$

The right-hand side has a saddle-point at $u=\alpha$ and a branch-point at $u=0$. Furthermore,

$$
\frac{t}{a c}-1 \sim u^{\alpha / y}, \quad \text { as } u \rightarrow 0 .
$$

We insist that the saddle-point at $t=S p_{+}$is mapped to $u=\alpha$. Hence, $\alpha$ is defined via

$$
f\left(S p_{+}\right)=\alpha(1-\ln \alpha)+\ln (a c)+y \ln (1-c),
$$

and the property that $\alpha \sim y$ as $y \rightarrow 0$. Since

$$
\frac{d t}{d u}=\frac{u-\alpha}{u f^{\prime}(t)}=\frac{t(t-a)(t-a c)(u-\alpha)}{\left(t-S p_{+}\right)\left(t-S p_{-}\right) u}
$$


it follows that

$$
\left.\frac{d t}{d u}\right|_{u=\alpha}=\frac{1}{\sqrt{\alpha f^{\prime \prime}\left(S p_{+}\right)}}
$$

We have

$$
\frac{1}{2 \pi i} \int_{\mathcal{C}_{ \pm}} \frac{G_{ \pm}(t)}{t^{n+1}} d t=\frac{e^{\mp x \pi i} K}{(a c)^{n}(1-c)^{n y} 2 \pi i} \int_{0}^{\infty} e^{-n(u-\alpha \ln u)} \tilde{g}_{0}(u) u^{\frac{\alpha}{y}(\gamma+1)-1} d u
$$

where

$$
\tilde{g}_{0}(u)=\tilde{g}(t) \frac{d t}{d u} u^{1-\frac{\alpha}{y}(\gamma+1)} .
$$

The power of $u$ in (7.10) is chosen such that $\tilde{g}_{0}(u)$ has no branch-point at $u=0$.

To obtain a uniform asymptotic expansion we have to expand $\tilde{g}_{0}(u)$ near $u=\alpha$. Hence,

$$
S_{n}(x) \sim \frac{-\sin (x \pi) K \tilde{g}_{0}(\alpha)}{(a c)^{n}(1-c)^{n y} \pi} n^{-\alpha n-\frac{\alpha}{y}(\gamma+1)} \Gamma\left(\alpha n+\frac{\alpha}{y}(\gamma+1)\right),
$$

as $n \rightarrow \infty$, uniformly for $y \in\left[0, Y_{-}-\varepsilon\right]$, where $\varepsilon$ is a small positive constant. Again we can see that $S_{n}(x)$ has zeros at approximately $x=m$, where $m$ is a bounded nonnegative integer. This is in agreement with the final paragraph of $\S 2$.

\section{The case $y \approx Y_{+}$}

When $y \approx Y_{+}$we have for the $\theta$ in (3.14) $\theta \approx \pi$. We will replace $\theta$ by $\pi-\varphi$ and the link between the new $\varphi$ and $y$ is now

$$
\cos \varphi=1+\frac{1-c}{2 \sqrt{c}}\left(y-Y_{+}\right), \quad \text { and take } \begin{array}{r}
y>Y_{+} \\
y<Y_{+}
\end{array} \begin{array}{r}
-\varphi i>0 \\
\varphi>0
\end{array}
$$

Hence, if $y \approx Y_{+}$then $\varphi \approx 0$.

In this case we use the cubic transformation

$$
f(t)=\frac{1}{3} u^{3}-\omega u+\psi .
$$

The right-hand side of (8.2) has saddle-points at $u=\mp \sqrt{\omega}$, and we will insist that these correspond to $t=S p_{ \pm}=a \sqrt{c} e^{(\pi \pm \varphi) i}$, respectively. This gives us

$$
\psi=\ln (a)+\frac{1}{2}(1+y) \ln (c)+i \pi(1-y),
$$

and

$$
\frac{2}{3} \omega^{3 / 2}=(1+y) \varphi i+y \ln \left(\frac{e^{-\varphi i}+\sqrt{c}}{e^{\varphi i}+\sqrt{c}}\right) .
$$

The reader can check that for the right-hand side in (8.4), we have

$$
(1+y) \varphi i+y \ln \left(\frac{e^{-\varphi i}+\sqrt{c}}{e^{\varphi i}+\sqrt{c}}\right) \sim \frac{2}{3} \frac{c^{-1 / 4}(1-\sqrt{c})^{2}}{\sqrt{1-c}}\left(y-Y_{+}\right)^{3 / 2},
$$


as $y \rightarrow Y_{+}$. Hence,

$$
\omega \sim \frac{c^{-1 / 6}(1-\sqrt{c})^{4 / 3}}{(1-c)^{1 / 3}}\left(y-Y_{+}\right), \quad \text { as } y \rightarrow Y_{+} .
$$

It is not difficult to show that on the interval $y \in\left(Y_{-}, \infty\right), \omega(y)$ is an increasing analytic function of $y$ with

$$
\omega\left(Y_{-}\right)=-\left(\frac{3 \pi \sqrt{c}}{1+\sqrt{c}}\right)^{2 / 3}, \quad \omega\left(Y_{+}\right)=0 .
$$

The local behaviour of transformation (8.2) near $t \approx a \sqrt{c} e^{(\pi-\varphi) i}$ and $u \approx \sqrt{\omega}$ is

$$
\frac{-i e^{2 \varphi i} \sin \varphi}{a^{2} \sqrt{c}(1-c) y}\left(t-a \sqrt{c} e^{(\pi-\varphi) i}\right)^{2} \approx \sqrt{\omega}(u-\sqrt{\omega})^{2},
$$

from which it follows that in the case $\omega<0$ we have to take $\sqrt{\omega}=-i \sqrt{|\omega|}$.

Using substitution (8.2) integral (5.2) becomes

$$
\frac{1}{2 \pi i} \int_{\mathcal{C}_{+}} \frac{\widetilde{G_{+}}(t)}{t^{n+1}} d t=\frac{e^{-x \pi i} K e^{-n \psi}}{2 \pi i} \int_{\infty}^{\infty e^{-2 \pi i / 3}} e^{-n\left(\frac{1}{3} u^{3}-\omega u\right)} g_{0}(u) d u,
$$

where

$$
g_{0}(u)=t^{-1} g(t) \frac{d t}{d u}=t^{-1} g(t) \frac{u^{2}-\omega}{f^{\prime}(t)} .
$$

The orientation of the $u$-integral in (8.9) follows when one takes the obvious squarehoods in (8.8). Note that from l'Hôpital's rule we obtain

$$
\left.\frac{d t}{d u}\right|_{u= \pm \sqrt{\omega}}=\sqrt{\frac{ \pm 2 \sqrt{\omega}}{f^{\prime \prime}\left(a \sqrt{c} e^{(\pi \mp \varphi) i}\right)}} .
$$

It follows that

$$
g_{0}( \pm \sqrt{\omega})=-\sqrt{\frac{i \sqrt{w}(1-c) y}{\sqrt{c} \sin \varphi}}\left(\frac{(1-c) y}{a c}\right)^{\gamma} \frac{\left(1+\sqrt{c} e^{\mp \varphi i}\right)^{1-\beta}}{1+a \sqrt{c} e^{\mp \varphi}} .
$$

Again, we substitute into (8.9)

$$
g_{0}(u)=p+q u+\left(u^{2}-\omega\right) h_{0}(u),
$$

where

$$
p=\frac{g_{0}(\sqrt{\omega})+g_{0}(-\sqrt{\omega})}{2}, \quad q=\frac{g_{0}(\sqrt{\omega})-g_{0}(-\sqrt{\omega})}{2 \sqrt{\omega}},
$$

and obtain

$$
\begin{aligned}
\frac{1}{2 \pi i} \int_{\mathcal{C}_{+}} \frac{\widetilde{G_{+}}(t)}{t^{n+1}} d t \sim \frac{-e^{-x \pi i} K e^{-n \psi}}{2 \pi i} & \left(p \int_{\infty e^{-2 \pi i / 3}}^{\infty} e^{-n\left(\frac{1}{3} u^{3}-w u\right)} d u\right. \\
& \left.+q \int_{\infty e^{-2 \pi i / 3}}^{\infty} u e^{-n\left(\frac{1}{3} u^{3}-w u\right)} d u\right) .
\end{aligned}
$$


Note that in this case $e^{-x \pi i} e^{-n \psi}=(-a)^{-n} c^{-(n+x) / 2}$. Using again the change variable $u=e^{-\pi i / 3} n^{-1 / 3} t$, we get

$$
\begin{array}{r}
\frac{1}{2 \pi i} \int_{\mathcal{C}_{+}} \frac{\widetilde{G_{+}}(t)}{t^{n+1}} d t \sim \frac{K(-a)^{-n}}{2 \pi i c^{(n+x) / 2}}\left(\frac{p e^{2 \pi i / 3}}{n^{1 / 3}} \int_{\infty e^{-\pi i / 3}}^{\infty e^{\pi i / 3}} e^{\frac{1}{3} t^{3}-w n^{2 / 3} e^{2 \pi i / 3} t} d t\right. \\
\left.+\frac{q e^{\pi i / 3}}{n^{2 / 3}} \int_{\infty e^{-\pi i / 3}}^{\infty e^{\pi i / 3}} t e^{\frac{1}{3} t^{3}-w n^{2 / 3} e^{2 \pi i / 3} t} d t\right)
\end{array}
$$

which is equivalent to,

$$
\begin{array}{r}
\frac{1}{2 \pi i} \int_{\mathcal{C}_{+}} \frac{\widetilde{G_{+}}(t)}{t^{n+1}} d t \sim \frac{K(-a)^{-n}}{c^{(n+x) / 2}}\left(\frac{p e^{2 \pi i / 3}}{n^{1 / 3}} \operatorname{Ai}\left(\omega e^{2 \pi i / 3} n^{2 / 3}\right)\right. \\
\left.-\frac{q e^{\pi i / 3}}{n^{2 / 3}} \operatorname{Ai}^{\prime}\left(\omega e^{2 \pi i / 3} n^{2 / 3}\right)\right)
\end{array}
$$

as $n \rightarrow \infty$. Hence,

$$
S_{n}(x) \sim \frac{2 K(-a)^{-n}}{c^{(n+x) / 2}} \Re\left\{\frac{p e^{2 \pi i / 3}}{n^{1 / 3}} \operatorname{Ai}\left(\omega e^{2 \pi i / 3} n^{2 / 3}\right)-\frac{q e^{\pi i / 3}}{n^{2 / 3}} \mathrm{Ai}^{\prime}\left(\omega e^{2 \pi i / 3} n^{2 / 3}\right)\right\},(8.1
$$

as $n \rightarrow \infty$. Using the fact that $p$ and $q$ are real-valued and the connection relation

$$
\operatorname{Ai}(\omega)+e^{-2 \pi i / 3} \operatorname{Ai}\left(\omega e^{-2 \pi i / 3}\right)+e^{2 \pi i / 3} \operatorname{Ai}\left(\omega e^{2 \pi i / 3}\right)=0,
$$

(see $(9.2 .12)$ in $[9])$, we get

$$
S_{n}(x) \sim \frac{K(-a)^{-n}}{c^{(n+x) / 2}}\left\{\frac{-p}{n^{1 / 3}} \mathrm{Ai}\left(\omega n^{2 / 3}\right)-\frac{q}{n^{2 / 3}} \mathrm{Ai}^{\prime}\left(\omega n^{2 / 3}\right)\right\},
$$

as $n \rightarrow \infty$, uniformly for $y \in\left[Y_{-}+\varepsilon, 1 / \varepsilon\right]$, where $\varepsilon$ is a small positive constant.

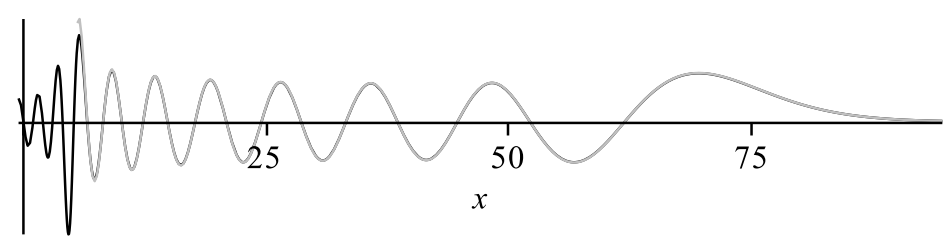

Fig. 4. The graph of a rescaled version of $S_{20}(x)$, where $a=\frac{5}{6}, c=\frac{1}{3}, \beta=\frac{9}{8}$ (black), and approximation (8.20) (grey). Note that only near $y=Y_{-}$the difference is visible.

\section{Large zeros}

Let $a_{k}$ be the $k^{t h}$ zero of the Airy function $\operatorname{Ai}(x)$ with $k=1,2,3, \cdots$. Thus $a_{1}=$ $-2.338 \cdots, a_{2}=-4.088 \cdots$ (See $\S 9.9$ in [9].) The dominant term in (8.20) includes the factor $\operatorname{Ai}\left(\omega n^{2 / 3}\right)$, and the zeros of this function are located at

$$
\omega_{k}=a_{k} n^{-2 / 3} .
$$


Hence, for fixed $k$ these will approach zero as $n \rightarrow \infty$, and we can use (8.6)

$$
a_{k} n^{-2 / 3} \sim \frac{1-\sqrt{c}}{(c+\sqrt{c})^{1 / 3}}\left(y_{n, k}-Y_{+}\right)
$$

as $n \rightarrow \infty$, where $x=n y_{n, j}$ are the zeros of $S_{n}(x)$ with

$$
0<y_{n, n}<y_{n, n-1}<\ldots<y_{n, 1}<\infty \text {. }
$$

Thus for the large zeros we obtain,

$$
y_{n, k} \sim Y_{+}+\frac{(c+\sqrt{c})^{1 / 3}}{1-\sqrt{c}} \frac{a_{k}}{n^{2 / 3}},
$$

as $n \rightarrow \infty$, where $k=1,2,3, \cdots$ is fixed.

To obtain a better approximation for the zeros we use both terms in the righthand side of (8.20). Its small zeros are located at $\omega_{k}=a_{k} n^{-2 / 3}+\delta$, where $\delta=$ $o\left(n^{-2 / 3}\right)$, as $n \rightarrow \infty$. We substitute this expression for $\omega$ into the right-hand side of $(8.20)$, approximate $\mathrm{Ai}\left(\omega_{k} n^{2 / 3}\right) \approx \delta n^{2 / 3} \mathrm{Ai}^{\prime}\left(a_{k}\right)$ and $\mathrm{Ai}^{\prime}\left(\omega_{k} n^{2 / 3}\right) \approx \operatorname{Ai}^{\prime}\left(a_{k}\right)$. Hence, $\delta \approx-q /(p n)$. From (8.14), (8.12) and (8.4) we can obtain the limit of $q / p$ as $\omega \rightarrow 0$. The result is the approximation

$$
\omega_{k} \sim a_{k} n^{-2 / 3}+\left(\frac{a}{1+a \sqrt{c}}-\frac{1-\beta}{1+\sqrt{c}}\right) \frac{(c+\sqrt{c})^{2 / 3}}{n}, \quad \text { as } n \rightarrow \infty .
$$

Hence,

$$
y_{n, k} \sim Y_{+}+\frac{(c+\sqrt{c})^{1 / 3}}{1-\sqrt{c}} \frac{a_{k}}{n^{2 / 3}}+\left(\frac{a}{1+a \sqrt{c}}-\frac{1-\beta}{1+\sqrt{c}}\right) \frac{c+\sqrt{c}}{(1-\sqrt{c}) n},
$$

as $n \rightarrow \infty$, where $k=1,2,3, \cdots$ is fixed.

When we let $\lambda \rightarrow 0$, that is, $a \rightarrow 1$, we obtain a two term approximation for the large zeros of the classical Meixner polynomials:

$$
y_{n, k} \sim Y_{+}+\frac{(c+\sqrt{c})^{1 / 3}}{1-\sqrt{c}} \frac{a_{k}}{n^{2 / 3}}+\frac{\beta \sqrt{c}}{(1-\sqrt{c}) n},
$$

as $n \rightarrow \infty$, where $k=1,2,3, \cdots$ is fixed. The first two terms in this approximation agree with the result (2.42) given in [6]. The third term appears to be a new term, and is surprisingly simple.

We finish with a numerical illustration. Taking $n=100$ and $k=1$ in (9.4) we obtain $y_{n, 1} \approx 3.4831614$, and from $(9.6)$ we obtain $y_{n, 1} \approx 3.4969920$. The 'exact' location is $y_{n, 1}=3.4999640$. The errors seem to be of the correct order.

\section{Acknowledgments}

The authors thank D. E. Dominici for suggesting the problem and one of the referees for helpful suggestions, which resulted in $\S 7$. 


\section{References}

[1] I. Area, E. Godoy, AND F. MARCELlán, Inner products involving differences: the Meixner-Sobolev polynomials, J. Differ. Equations Appl., 6 (2000), pp. 1-31.

[2] I. Area, E. Godoy, F. Marcellán, and J. J. Moreno-Balcázar, Ratio and Plancherel-Rotach asymptotics for Meixner-Sobolev orthogonal polynomials, J. Comput. Appl. Math., 116 (2000), pp. 63-75.

[3] N. Bleistein, Uniform asymptotic expansions of integrals with stationary point near algebraic singularity, Comm. Pure Appl. Math., 19 (1966), pp. 353-370.

[4] C. Chester, B. Friedman, and F. Ursell, An extension of the method of steepest descents, Proc. Cambridge Philos. Soc., 53 (1957), pp. 599-611.

[5] H. W. Нетнсоте, Error bounds for asymptotic approximations of zeros of transcendental functions, SIAM J. Math. Anal., 1 (1970), pp. 147-152.

[6] X.-S. Jin And R. Wong, Asymptotic formulas for the zeros of the Meixner polynomials, J. Approx. Theory, 96 (1999), pp. 281-300.

[7] J. Moreno-Balcázar, T. PÉrez, And M. Piñar, A generating function for nonstandard orthogonal polynomials involving differences: The Meixner case, Ramanujan J., 25 (2011), pp. 21-35.

[8] A. B. Olde DaAlhuis, Hypergeometric function, in NIST handbook of mathematical functions, U.S. Dept. Commerce, Washington, DC, 2010, pp. 383-401.

[9] F. W. J. Olver, Airy and related functions, in NIST handbook of mathematical functions, U.S. Dept. Commerce, Washington, DC, 2010, pp. 193-213.

[10] F. W. J. Olver And R. Wong, Asymptotic approximations, in NIST handbook of mathematical functions, U.S. Dept. Commerce, Washington, DC, 2010, pp. 41-70. 